\title{
The role of endobronchial ultrasound (EBUS) in radiographically occult mediastinal disease and the future of EBUS
}

\author{
Maren Schuhmann • Mark G. H. Bryant • \\ Felix J. F. Herth • Daniela Gompelmann
}

Published online: 25 January 2012

(C) Springer Science+Business Media, LLC 2012

\begin{abstract}
Lung cancer remains a common cancer worldwide. The normal mediastinum on imaging often requires further clarification prior to potentially curative surgery. In this article we review the indications for endobronchial ultrasound (EBUS) and compare it with radiological imaging. We evaluate recent publications on EBUS as a means to evaluate $\mathrm{CT}$ and PET negative mediastinal lymph nodes. We also discuss the role of a combined approach of oesophageal ultrasound (EUS) with EBUS for staging of the mediastinum and evaluate the future of EBUS in lung cancer. Transbronchial needle forceps biopsy is a new approach to obtain a larger histological specimen under ultrasound guidance and may be used for immunohistochemistry analysis. In the future EBUS may also offer treatment options as outlined in this article.
\end{abstract}

M. Schuhmann ( $₫)$

University Hospital Southampton,

Tremona Road,

Southampton SO16 6YD, UK

e-mail: mschuhm@hotmail.com

M. G. H. Bryant

Royal Bournemouth Hospital,

Castle Lane East,

Bournemouth BH77DW, UK

e-mail:mghbryant@gmail.com

F. J. F. Herth · D. Gompelmann

Thoraxklinik Heidelberg,

Amalienstr. 5,

69126 Heidelberg, Germany

F. J. F. Herth

e-mail: felix.herth@thoraxklinik-heidelberg.de

D. Gompelmann

e-mail: daniela.gompelmann@thoraxklinik-heidelberg.de
Keywords Endobronchial ultrasound · Bronchoscopy Lung cancer - Endoscopic ultrasound · Mediastinal disease . Transbronchial needle forceps

\section{Introduction}

Lung cancer remains one the most common cancers worldwide and on diagnosis is often already at an advanced stage. In $26 \%$ of cases mediastinal lymph nodes are involved at the time of diagnosis and in $49 \%$ extrathoracic metastases can be found. In order to determine adequate treatment it is crucial to accurately stage non-small cell lung cancer (NSCLC). Computer tomography is often the first staging procedure to take place in disease evaluation. Other staging procedures include positron electron tomography (PET) or PET-CT scanning. Over the last few years endobronchial as well as esophageal ultrasound have been used to assess for tumor invasion, mediastinal lymph node enlargement, and also to sample suspicious nodes. International guidelines still recommend mediastinoscopy as the gold standard for staging of the mediastinum, although combining EBUS and EUS has shown very high sensitivity and specificity for the detection of mediastinal metastases.

\section{Radiology}

In most centers the standard staging procedure consists of CT scanning to determine local tumor extension as well as nodal and distant metastases. Lymph nodes are considered abnormal if their short-axis diameter is $>1 \mathrm{~cm}$, but smaller lymph nodes may contain metastatic material and lymph nodes may be enlarged due to benign disease.

The accuracy of CT scanning in the diagnosis of mediastinal disease is low, largely as a result of reliance on nodal size. In 
one study $20 \%$ of nodes thought to be positive were benign [1], the authors suggesting that this is not suitable for staging. The sensitivity of CT for the detection of mediastinal lymph node spread is approximately $50 \%$ with a specificity of $85 \%[2 \cdot \bullet]$.

Surgical studies have previously shown metastatic lymph nodes to be present at the time of surgery in $15 \%-20 \%$ of CT-negative patients [3-5].

Morphologically normal nodes have been shown to contain metastatic disease. The introduction of functional imaging in PET allowed for the assessment of the metastatic disease activity, therefore increasing the sensitivity of imaging by identifying the involved small node $<1 \mathrm{~cm}$, but also excluding from staging the larger nodes that did not contain metastatic disease. PET has been found to be superior to CT scanning in determining mediastinal nodal involvement. PET was accurate in $94 \%$ of patients in characterizing " $N$ " disease as compared to $61 \%$ with CT [6]. This was also shown in several meta-analyses, showing that PET was more accurate and more specific in predicting mediastinal node involvement $[7,8]$. Spatial resolution of PET is poor, however, and lesions of less than $5 \mathrm{~mm}$ may be falsely negative due to the limited resolution of PET scanning as well as volume effects [9].

The introduction of combined CT and PET imaging brought simultaneous morphological and functional analysis. Integrated PET-CT improves the diagnostic accuracy of the staging of non-small cell lung cancer. In a study by Lardinois et al. [10], PET-CT added additional information in $41 \%$ of patients beyond that provided by visual correlation of PET and CT.

Despite these advances, a recent meta analysis has shown overall sensitivity for PET/CT to be $76 \%$ and specificity $88 \%$ [11]. PET-CT assessment of the mediastinum is associated with a clinically relevant false-positive rate.

\section{Mediastinoscopy}

Mediastinoscopy is to date still considered the gold standard for mediastinal staging by various societies and has a sensitivity of approximately $78 \%$ [12]. However, this is an invasive procedure, which requires general anesthesia and only certain lymph node stations are accessible (lymph node stations 2, 4, and anterior 7). The reported morbidity for this procedure is $2 \%$ and mortality $0.08 \%$ [13]. Furthermore, undetected mediastinal metastases often lead to unnecessary thoracotomies [14].

\section{Endobronchial ultrasound}

Endobronchial ultrasound (EBUS) provides visualization of the tracheobronchial wall and para-bronchial structures and has become a routinely performed procedure in diagnostic bronchoscopy. It plays an important part in the staging of lung cancer patients in particular. EBUS can be used in this setting for the exclusion of local infiltration in case of early lung cancer, for detection of tumor infiltration of the tracheobronchial wall or mediastinal structures, as well as for the detection of lymph node involvement in lung cancer patients [15].

Endosonography was initially used in the field of gastrointestinal endoscopy, especially for the staging of tumors of the esophagus and cardia [16]. Several studies in as early as 1990 showed that endoscopic ultrasonography is more accurate in staging patients with gastrointestinal carcinoma compared to radiological imaging procedures alone $[16,17]$. To try and imitate these results, the use of ultrasonography within the airways was developed and over the last decades EBUS has been widely introduced with great success.

Nowadays, two different EBUS techniques for the staging of lung cancer can be distinguished. Radial balloon probes provide a view of the multilayer structure of the tracheobronchial wall and adjacent structures, whereas ultrasound-guided transbronchial needle aspiration allows sampling of enlarged hilar or mediastinal lymph nodes.

Involvement of tracheobronchial wall and mediastinal tumor infiltration

Endobronchial ultrasound plays an important role in the evaluation of the involvement of the tracheobronchial wall. At the tip of the radial endobronchial ultrasound probe, a water-inflatable balloon provides contact to the tracheobronchial wall and thus allowing sound wave transmission.

Particularly in cases of suspicious mucosal lesions, which are not visible on radiological imaging, the evaluation of depth penetration by EBUS is a crucial factor to distinguish between noninvasive and invasive carcinoma. Furthermore, it was shown that EBUS is a highly accurate diagnostic tool and superior to chest $\mathrm{CT}$ in evaluating airway involvement of central intrathoracic tumors [18]. In this prospective trial, 105 patients with central thoracic malignancies underwent computed tomography followed by EBUS evaluation and subsequent surgical procedure. The histopathologic findings after surgery revealed an accuracy of $94 \%$ for EBUS. Chest CT was far inferior with an accuracy of only $51 \%$.

Hence EBUS provides more accurate local tumor staging compared to radiological imaging procedures and thus improving the base for rational therapy decision.

Lymph node involvement

Endobronchial ultrasound transbronchial needle aspiration (EBUS-TBNA) is a relatively recent development, which is performed by using an EBUS bronchoscope and was first reported by Krasnik et al. [19] in 2003 and by Yasufuku et 
al. [20] in 2004. EBUS bronchoscopes currently available have an outer diameter of $6.3-6.9 \mathrm{~mm}$ with a $2.0 \mathrm{~mm}$ instrument channel and a $30^{\circ}$ side viewing optic. The convex array ultrasound transducer is mounted at the distal tip of the bronchoscope and covered with a water-inflatable balloon. Scanning is performed at a frequency of $7.5-12 \mathrm{MHz}$ and achieves a tissue penetration of $20-50 \mathrm{~mm}$. A 21 or 22 gauge needle, that contains an internal stylet, can then be advanced via the working channel into the lymph nodes under direct ultrasound control to sample the lymph node. The internal stylet avoids sampling tissue from the bronchial wall and stabilizes the needle. After puncture of the bronchial wall, the stylet is removed. Then, suction is applied at the proximal end of the needle [21] (Fig. 1).

EBUS can access high mediastinal (lymph node station 1), upper paratracheal (station 2), lower paratracheal (station 4), the subcarinal level (station 7), as well as the hilar lymph nodes (station 10 and 11).

In contrast to mediastinoscopy the procedure can be performed under minor sedation and does not require theatre time. It is safe and carries a very low morbidity.

EBUS has been used for several years and has been shown to be a safe method for sampling mediastinal nodes under real-time sonography. It is used as an important tool in diagnosing lymphadenopathy due to sarcoidosis, tuberculosis, thymoma, lymphoma, or metastases of extrathoracic malignancies, but it has become a routinely performed bronchoscopic procedure in mediastinal staging of lung cancer. Lymph nodes with a size of $5 \mathrm{~mm}$ and upwards can be biopsied and have shown excellent diagnostic yield.

Several meta-analyses have been published on the accuracy of EBUS staging. One review included 10 trials which focused on mediastinal lymph node staging by EBUS-TBNA in 817 patients and revealed an overall specificity of $100 \%$ and a

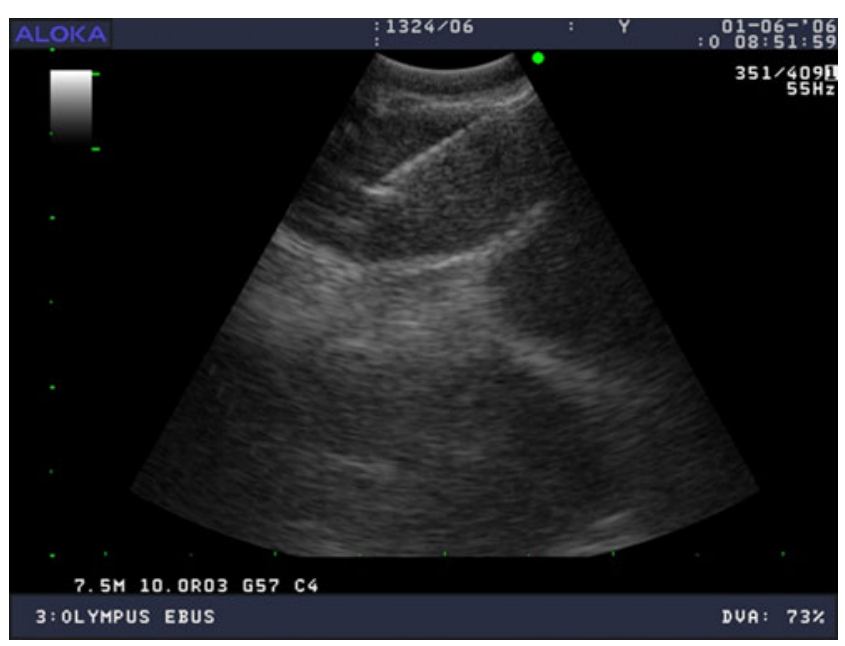

Fig. 1 Endobronchial ultrasound with needle in enlarged lymph node sensitivity of $88 \%$ [22]. Another meta-analysis from 2009 included 1,299 patients and showed a pooled sensitivity for EBUS of $93 \%$ [23•].

EBUS-TBNA has also been reported as a diagnostic tool for the evaluation of the radiologically normal mediastinum [24]. In order to determine the sensitivity and specificity of EBUS in a radiologically normal mediastinum, Herth et al. [24] examined 100 patients with a lung mass (T1 to T4) but radiologically normal (N0) mediastinum on $\mathrm{CT}$ criteria. In these patients, 119 lymph nodes with a mean diameter of $8.1 \mathrm{~mm}$ were detected by endobronchial ultrasound and sampled. All patients had a least one node biopsied and all underwent mediastinoscopy or thoracotomy following this. The authors identified malignant mediastinal lymph nodes on EBUS that had been negative on CT scanning in 19 out of 100 patients. The sensitivity of EBUS-TBNA for detecting malignancy was $92.3 \%$, specificity $100 \%$, and the negative predictive value was $96.3 \%$ in this study. However, as neither PET nor PET-CT were included in the guidelines at the time of this study, these patients had only been radiologically staged by means of CT scanning.

Yasufuku et al. [25] in 2006 examined 102 patients with proven or suspected lung cancer and all patients underwent CT, PET, and EBUS-TBNA. Comparison was made of EBUS-TBNA staging with CT and PET in these potentially operable patients. CT scanning in this study for the prediction of mediastinal lymph node involvement showed a sensitivity of $76.9 \%$, a specificity of $55.3 \%$, negative predictive value of $87.5 \%$, and an accuracy of $60.8 \%$. PET identified several false-positive lymph nodes and the sensitivity was $80 \%$, specificity $70.1 \%$, and accuracy $72.5 \%$. In comparison to these techniques, EBUS was significantly more able to predict the correct lymph node status and was highly sensitive and specific. The sensitivity of EBUS-TBNA for the prediction of mediastinal lymph node involvement was $92.3 \%$, specificity was $100 \%$, and accuracy $98 \%$.

A further study by Herth et al. [26•] was undertaken in 2008 to investigate with endobronchial ultrasound, CT, and PET negative mediastinal lymph nodes in patients with lung cancer. A total of 100 patients with a high suspicion for NSCLC, but who had no lymph node enlargement on $\mathrm{CT}$ and a negative PET scan, underwent endobronchial ultrasound. Lymph nodes were biopsied and patients subsequently went for surgical staging to confirm the EBUS findings. EBUS-TBNA in this study had a sensitivity for the detection of malignancy of $89 \%$, specificity of $100 \%$, and a negative predictive value of $98.9 \%$.

Rapid on-site evaluation of the samples obtained during an EBUS procedure has been shown to increase the yield of these procedures further as well as being cost effective [27]. This involves a cytopathologist screening the samples in the endoscopy suite for either presence or absence of diagnostic material. This way it can be ensured that diagnostic samples are obtained prior to ending the procedure and also avoids 
unnecessary multiple punctures once a diagnostic sample has been obtained.

\section{Limitations of EBUS}

Despite its clear value in the diagnosis of occult as well as in indeterminate mediastinal involvement, EBUS-TBNA still has its limitations. One of these is the lack of access to the paraesophageal and subaortic lymph nodes (position 5, 6, 8, and 9). Therefore, an accurate complete endoscopic mediastinal staging of lung cancer can only be achieved by combining the two complementary methods: EBUS-TBNA and esophageal ultrasound-guided fine-needle aspiration (EUS-FNA) [28].

\section{Combined approach with EBUS and EUS}

The combined approach offers the so-called complete mediastinal staging. This way nearly all lymph node stations within the mediastinum can be accessed, hence increasing the sensitivity for mediastinal staging. A randomized trial of 241 patients by Annema et al. [29••] directly compared mediastinoscopy with endosonography for mediastinal nodal staging. Either combined EUS/EBUS needle aspiration followed by mediastinoscopy (if endosonography negative) or mediastinoscopy alone was performed on patients who had enlarged mediastinal nodes by CT criteria or showed uptake on PET. This was then followed by thoracotomy with complete lymph node dissection if no mediastinal disease was present. The results indicated that mediastinal staging with endosonography followed by mediastinoscopy improved the detection of mediastinal metastases significantly $(94 \%$ vs. $79 \%$ with surgical staging alone) and reduced the number of unnecessary thoracotomies. The results raised the question if the combined approach might replace mediastinoscopies in the future given its lower risk and cost profile.

In another study, it could be demonstrated that the combined approach of EBUS-TBNA and EUS-FNA could be performed by one single linear ultrasound bronchoscope [30]. In 139 patients with lung cancer, 619 lymph nodes were biopsied by either EUS-FNA or EBUS-TBNA. EBUS-TBNA was performed first, followed by EUS-FNA with the same bronchoscope. Sensitivity was $89 \%$ for EUS-FNA and $92 \%$ for EBUS-TBNA. The combined approach showed a sensitivity of $96 \%$.

Szlubowski et al. [31] performed the combined approach in 120 patients with a radiologically normal mediastinum. The overall sensitivity for the combined approach was $68 \%$, the negative predictive value was $91 \%$, and the prevalence of $2 / 3$ disease was recorded at $22 \%$. The combined needle aspiration approach was shown to have a higher diagnostic yield than EBUS or EUS-needle aspiration alone.

\section{Future of EBUS}

One disadvantage of TBNA is that the cytological specimens obtained with 21 or 22 gauge needle are limited and therefore sometimes insufficient to provide a firm diagnosis. In particular, increasingly important immunohistochemistry and mutational analysis, used to determine targeted therapy in lung cancer patients, are commonly not possible due to sample size. Therefore, new methods providing larger tissue samples for histological examination are presently being developed. One of these approaches is the sampling of tissue by a transbronchial needle-forceps (TBNF) that has been developed for the EBUS bronchoscope. This new instrument combines the features of a needle with that of a forceps. A sharp tip allows penetration of the bronchial wall and the jaws can then be opened and closed within the node to obtain larger tissue samples. A recent pilot study of EBUS-guided lymph node biopsy with a TBNF [32] involved 50 patients with enlarged lymph nodes greater than $15 \mathrm{~mm}$ in short axis diameter on CT or PET-positive lymph nodes. All patients underwent EBUSguided TBNF of mediastinal as well as hilar lymph nodes. In $86 \%$ of patients, TBNF provided a histological diagnosis including malignancies as well as benign diseases. In all patients with a diagnosis of non-small cell lung cancer $(n=$ 24), immunohistochemistry analysis of the specimen provided a specific subtype. The sensitivity, specificity, positive predictive value, negative predictive value, and accuracy value for the sampling technique was $88 \%, 100 \%, 100 \%, 17 \%$, and $88 \%$, respectively.

In the future, it seems that the EBUS scope will not only be used as a diagnostic tool, but also for therapeutic procedures. In 2006, a review related to transbronchial needle injection (TBNI) was published [33]. The authors described the therapeutic as well as diagnostic applications of TBNI that were performed by using a conventional TBNA needle without simultaneous ultrasonography guidance. Therapeutic strategies consisted of treatment of lung cancer and thoracic malignancies by local injection of ethanol [34], tumor suppressor chemotherapeutic agents [35, 36], and immunostimulatory genes [37]. TBNI also offers treatment for endobronchial inflammatory lesions [38], recurrent respiratory papillomatosis [39], and bronchopleural fistulas [40]. In addition to these therapeutic strategies, TBNI can be used for identifying the sentinel lymph node by injection of radiolabeled technetium- $99 \mathrm{~m}$ into the visible endobronchial tumor or into the subsegment visible proximal to the tumor [41]. Furthermore, TBNI can be utilized to guide surgical localization of small pulmonary nodules by injection of the visceral pleura with indigo carmine dye [42].

The technique of endobronchial or transbronchial injection of various agents has already been reported in the last decades, but it has not been widely used. In the future, these approaches can be improved by using EBUS-guided transbronchial needle injections, thus providing a more accurate instillation of 
the different agents into a specific predetermined target. Therefore, EBUS-guided TBNI will broaden this therapeutic approach in the future and will extend the therapeutic bronchoscopic spectrum.

\section{Conclusions}

The radiologically negative mediastinum in lung cancer patients remains a diagnostic problem. Endobronchial ultrasound is a minimally invasive procedure, which not only identifies potentially metastatic lymph nodes but also offers the possibility of sampling the lymph nodes. In the radiologically normal mediastinum, EBUS has been shown to be of great use to identify malignant nodes. Its sensitivity and specificity far exceeds conventional radiological means of staging. International guidelines still favor mediastinoscopy as the gold standard to determine mediastinal involvement. The sensitivity of EBUS and EUS in combination however is such that it may replace more invasive staging in the future.

EBUS is ever developing and has the potential of not only offering diagnostic interventions but has already been shown to have its use as a therapeutic tool. This field will certainly expand over the next few years.

Disclosure No potential conflicts of interest relevant to this article were reported.

\section{References}

Papers of particular interest, published recently, have been highlighted as:

- Of importance

•. Of major importance

1. McLoud TC. "A system for the clinical staging of lung cancer"- $\mathrm{a}$ commentary. AJR Am J Roentgenol. 2006;187:269-70.

2. •- Silvestri GA, Gould MK, Margolis ML, et al. Noninvasive staging of non-small cell lung cancer: ACCP evidenced-based clinical practice guidelines (2nd edition). Chest. 2007;132:178S201S. Major reference guidelines with an overview of evidence for noninvasive staging.

3. Hoffmann H. Invasive staging of lung cancer by mediastinoscopy and video-assisted thoracoscopy. Lung Cancer. 2001;34 Suppl 3: S3-5.

4. Luke WP, Pearson FG, Todd TR, et al. Prospective evaluation of mediastinoscopy for assessment of carcinoma of the lung. J Thorac Cardiovasc Surg. 1986;91:53-6.

5. Coughlin M, Deslauriers J, Beaulieu M, et al. Role of mediastinoscopy in pretreatment staging of patients with primary lung cancer. Ann Thorac Surg. 1985;40:556-60.

6. Gupta NC, Graeber GM, Bishop HA. Comparative efficacy of positron emission tomography with fluorodeoxyglucose in evaluation of small $(<1 \mathrm{~cm})$, intermediate $(1$ to $3 \mathrm{~cm})$, and large $(>3 \mathrm{~cm})$ lymph node lesions. Chest. 2000;117:773-8.
7. Birim O, Kappetein AP, Stijnen T, et al. Meta-analysis of positron emission tomographic and computed tomographic imaging in detecting mediastinal lymph node metastases in nonsmall cell lung cancer. Ann Thorac Surg. 2005;79:375-82.

8. Gould MK, Kuschner WG, Rydzak CE, et al. Test performance of positron emission tomography and computed tomography for mediastinal staging in patients with non-small-cell lung cancer: a meta-analysis. Ann Intern Med. 2003;139:879-92.

9. Bousson V, Moretti JL, Weinmann P, et al. Assessment of malignancy in pulmonary lesions: FDG dual-head coincidence gamma camera imaging in association with serum tumor marker measurement. J Nucl Med. 2000;41:1801-7.

10. Lardinois D, Weder W, Hany TF, et al. Staging of non-small-cell lung cancer with integrated positron-emission tomography and computed tomography. N Engl J Med. 2003;348:2500-7.

11. Lv YL, Yuan DM, Wang K, et al. Diagnostic performance of integrated positron emission tomography/computed tomography for mediastinal lymph node staging in non-small cell lung cancer: a bivariate systematic review and meta-analysis. J Thorac Oncol. 2011;6:1350-8.

12. Detterbeck FC, Jantz MA, Wallace M, et al. Invasive mediastinal staging of lung cancer: ACCP evidence-based clinical practice guidelines (2nd edition). Chest. 2007;132:202S-20S.

13. Kiser AC, Detterbeck FC. General aspects of surgical treatment. Philadelphia: WB Saunders; 2001.

14. Fischer B, Lassen U, Mortensen J, et al. Preoperative staging of lung cancer with combined PET-CT. N Engl J Med. 2009;361:32-9.

15. Herth F, Becker HD. Endobronchial ultrasound of the airways and the mediastinum. Monaldi Arch Chest Dis. 2000;55:36-44.

16. Ziegler K, Sanft C, Semsch B, et al. Endosonography is superior to computed tomography in staging tumors of the esophagus and cardia. Gastroenterology Suppl. 1988;A517.

17. Ziegler K, Sanft C, Zimmer T, et al. Comparison of computed tomography, endosonography, and intraoperative assessment in TN staging of gastric carcinoma. Gut. 1993;34:604-10.

18. Herth F, Ernst A, Schulz M, et al. Endobronchial ultrasound reliably differentiates between airway infiltration and compression by tumor. Chest. 2003;123:458-62.

19. Krasnik M, Vilmann P, Larsen SS, et al. Preliminary experience with a new method of endoscopic transbronchial real time ultrasound guided biopsy for diagnosis of mediastinal and hilar lesions. Thorax. 2003;58:1083-6.

20. Yasufuku K, Chhajed PN, Sekine Y, et al. Endobronchial ultrasound using a new convex probe: a preliminary study on surgically resected specimens. Oncol Rep. 2004;11:293-6.

21. Herth F. Transbronchial needle aspiration and endobronchial ultrasound. Eur Respir Monogr. 2010;48:45-58.

22. Adams K, Shah PL, Edmonds L, et al. Test performance of endobronchial ultrasound and transbronchial needle aspiration biopsy for mediastinal staging in patients with lung cancer: systematic review and meta-analysis. Thorax. 2009;64:757-62.

23. • Gu P, Zhao YZ, Jiang LY, et al. Endobronchial ultrasound-guided transbronchial needle aspiration for staging of lung cancer: a systematic review and meta-analysis. Eur J Cancer. 2009;45:1389-96. Very clear meta-analysis as a point of reference.

24. Herth FJ, Ernst A, Eberhardt R, et al. Endobronchial ultrasoundguided transbronchial needle aspiration of lymph nodes in the radiologically normal mediastinum. Eur Respir J. 2006;28:910-4.

25. Yasufuku K, Nakajima T, Motoori K, et al. Comparison of endobronchial ultrasound, positron emission tomography, and CT for lymph node staging of lung cancer. Chest. 2006;130:710-8.

26. - Herth FJ, Eberhardt R, Krasnik M, et al. Endobronchial ultrasound-guided transbronchial needle aspiration of lymph nodes in the radiologically and positron emission tomography-normal mediastinum in patients with lung cancer. Chest. 2008;133:887-91. One of the first to evaluate EBUS in the normal mediastinum. 
27. Baram D, Garcia RB, Richman PS. Impact of rapid on-site cytologic evaluation during transbronchial needle aspiration. Chest. 2005;128:869-75.

28. Vilmann P, Krasnik M, Larsen SS, et al. Transesophageal endoscopic ultrasound-guided fine-needle aspiration (EUS-FNA) and endobronchial ultrasound-guided transbronchial needle aspiration (EBUS-TBNA) biopsy: a combined approach in the evaluation of mediastinal lesions. Endoscopy. 2005;37:833-9.

29. • Annema JT, van Meerbeeck JP, Rintoul RC, et al. Mediastinoscopy vs endosonography for mediastinal nodal staging of lung cancer: a randomized trial. JAMA. 2010;304:2245-52. This study shows very good results of combined staging with a potential to replace mediastinoscopy.

30. Herth FJ, Krasnik M, Kahn N, et al. Combined endoscopicendobronchial ultrasound-guided fine-needle aspiration of mediastinal lymph nodes through a single bronchoscope in 150 patients with suspected lung cancer. Chest. 2010;138:790-4.

31. Szlubowski A, Zielinski M, Soja J, et al. A combined approach of endobronchial and endoscopic ultrasound-guided needle aspiration in the radiologically normal mediastinum in non-small-cell lung cancer staging - a prospective trial. Eur J Cardiothorac Surg. 2010;37:1175-9.

32. Herth F, Schuler H, Gompelmann D, et al. EBUS-guided lymph node biopsy (EBUS-TBNB) with a transbronchial needle forceps (TBNF) - a pilot study. Eur Respir J. 2011.

33. Seymour CW, Krimsky WS, Sager J, et al. Transbronchial needle injection: a systematic review of a new diagnostic and therapeutic paradigm. Respiration. 2006;73:78-89.
34. Fujisawa T, Hongo H, Yamaguchi $\mathrm{Y}$, et al. Intratumoral ethanol injection for malignant tracheobronchial lesions: a new bronchofiberscopic procedure. Endoscopy. 1986;18:188-91.

35. Wagai F, Kinoshita M, Shiraki R, et al. The direct injection of several anti-cancer drugs into the primary lung cancer lesion through a fiberoptic bronchoscope (author's transl). Nihon Kyobu Shikkan Gakkai Zasshi. 1982;20:170-5.

36. Celikoglu F, Celikoglu SI. Intratumoural chemotherapy with 5fluorouracil for palliation of bronchial cancer in patients with severe airway obstruction. J Pharm Pharmacol. 2003;55:1441-8.

37. Roth JA, Nguyen D, Lawrence DD, et al. Retrovirus-mediated wild-type p53 gene transfer to tumors of patients with lung cancer. Nat Med. 1996;2:985-91.

38. Langford CA, Sneller MC, Hallahan CW, et al. Clinical features and therapeutic management of subglottic stenosis in patients with Wegener's granulomatosis. Arthritis Rheum. 1996;39:1754-60.

39. Milczuk HA. Intralesional cidofovir for the treatment of severe juvenile recurrent respiratory papillomatosis: long-term results in 4 children. Otolaryngol Head Neck Surg. 2003;128:788-94.

40. Varoli F, Roviaro G, Grignani F, et al. Endoscopic treatment of bronchopleural fistulas. Ann Thorac Surg. 1998;65:807-9.

41. Lardinois D, Brack T, Gaspert A, et al. Bronchoscopic radioisotope injection for sentinel lymph-node mapping in potentially resectable non-small-cell lung cancer. Eur J Cardiothorac Surg. 2003;23:824-7.

42. Endo M, Kotani Y, Satouchi M, et al. CT fluoroscopy-guided bronchoscopic dye marking for resection of small peripheral pulmonary nodules. Chest. 2004;125:1747-52. 